\title{
IRREGULARITIES OF DISTRIBUTION. V
}

WOLFGANG M. SCHMIDT

ABSTRACT. It is shown that for any finite or countable number of points with positive weights on the sphere, there is always a spherical cap such that the sum of the weights of the points in the cap is rather different from the expected value.

1. Introduction. In the last three papers [3], [4], [5] of the present series a method was developed to estimate irregularities of the distribution of points in Euclidean space or on the sphere. In these papers essential use was made of the fact that the number of points in any given set is always an integer. Hence it would appear that the method of these papers is not adaptable to points with weights. On the other hand, the method of Roth [2] is immediately adaptable to points with weights. Professor Roth therefore suggested the problem of generalizing the present method to deal with points with weights.

In fact it turns out that all the results of the three preceding papers of this series can be generalized in this direction. Moreover it is possible to deal with infinitely many points whose weights have a finite sum. In this note we shall restrict ourselves to Theorem B of [5], i.e. we shall deal only with the problem of spherical caps.

Let $S=S^{n}$ be the sphere consisting of points $x=\left(x_{1}, \cdots, x_{n}, x_{n+1}\right)$ with $x_{1}^{2}+\cdots+x_{n}^{2}+x_{n+1}^{2}=1$. Let $p_{1}, p_{2}, \cdots$ be points on $S$, with nonnegative weights $w_{1}, w_{2}, \cdots$ respectively. Assume throughout that

$$
\sigma=\sum_{i=1}^{\infty} w_{i}
$$

is positive and finite. Given a subset $\boldsymbol{A}$ of $S$, put

$$
\nu(A)=\sum_{p_{i} \in A} w_{i}
$$

In particular, $\nu(S)=\sigma$. Let $d_{S} \mathbf{x}$ be the canonical volume element on $S$, normalized so that $\int_{S} d_{S} x=1$. Given a measurable subset $\boldsymbol{A}$ of $S$, write $\mu(\boldsymbol{A})=\int_{A} d_{S} x$ and

$$
\Delta(\boldsymbol{A})=\nu(S) \mu(\boldsymbol{A})-\nu(\boldsymbol{A}) .
$$

Received by the editors December 12, 1969.

AMS Subject Classifications. Primary 1033; Secondary 5240.

Key Words and Phrases. Uniform distribution, irregularities of distribution, points on a sphere. 
Let $\mathbf{C}(r, \mathrm{c})$ be the spherical cap on $S$ with center $\mathrm{c}$ and with radius $r$. We shall deal only with caps whose radius does not exceed $\pi / 2$. Write

$$
\begin{gathered}
\Delta(r, \mathrm{c})=\Delta(\boldsymbol{C}(r, \mathrm{c})), \\
E(r, s)=\int_{S} \Delta(r, \mathrm{c}) \Delta(s, c) d_{S} \mathrm{c} .
\end{gathered}
$$

For any real $\alpha \geqq 1$, put

$$
\sigma(\alpha)=\sum_{i=1}^{N} w_{i}^{\alpha}
$$

Then $\sigma(1)=\nu(S)=\sigma$, and we have always $0<\sigma(\alpha)<\infty$.

Theorem. Suppose $n>1$ and $\epsilon>0$. Then

$$
\int_{0}^{\pi / 2} r^{-1} E(r, r) d r \gg \sigma\left(2+\frac{1}{n}+\epsilon\right) \sigma(1)^{-(1 / n)-\epsilon} .
$$

(Here and throughout, the constants implied by $\ll$ as well as constants $c_{1}, c_{2}, \cdots$ will depend only on $n$ and on $\epsilon_{\text {.) }}$

If $w_{N+1}=w_{N+2}=\cdots=0$, then we are really dealing with only $N$ points $\boldsymbol{p}_{1}, \cdots, \boldsymbol{p}_{N}$. Note that in this case the right-hand side of (5) exceeds

$$
\sigma(2) N^{-(1 / n)-\epsilon}=\left(w_{1}^{2}+\cdots+w_{N}^{2}\right) N^{-(1 / n)-\epsilon} .
$$

Namely, by Theorem 16 of [1], we have $(\sigma(s) / N)^{1 / s} \geqq(\sigma(r) / N)^{1 / r}$ if $s \geqq r>0$. Hence if $\alpha>0$ we have $\sigma(2+\alpha) / N \geqq(\sigma(2) / N)^{1+(\alpha / 2)}$ $\geqq(\sigma(2) / N)(\sigma(1) / N)^{\alpha}$, and applying this with $\alpha=(1 / n)+\epsilon$ we get $\sigma(2+(1 / n)+\epsilon) \geqq \sigma(2) \sigma(1)^{(1 / n)+\epsilon} N^{-(1 / n)-\epsilon}$.

Also note that if moreover $w_{1}=\cdots=w_{N}=1$, then $\sigma(\alpha)=N$ for every $\alpha$, and the right-hand side of (5) becomes $N^{1-(1 / n)-\epsilon}$.

Corollary. Suppose $n>1$ and $\epsilon>0$. There is a cap $\mathbf{C}(r, c)$ with

$$
\begin{aligned}
|\Delta(r, \mathrm{c})| \gg & \sigma\left(2+\frac{1}{n}+\epsilon\right)^{1 / 2} \sigma^{(-1 / 2 n)-(\epsilon / 2)} \\
& \cdot\left(1+\log \left(\frac{\sigma^{2+(1 / n)+\epsilon}}{\sigma\left(2+\frac{1}{n}+\epsilon\right)}\right)\right)^{-1 / 2} .
\end{aligned}
$$

In particular, if $w_{N+1}=w_{N+2}=\cdots=0$, then 


$$
\frac{\sigma^{2+(1 / n)+\epsilon}}{\sigma\left(2+\frac{1}{n}+\epsilon\right)} \leqq N^{2+(1 / n)+\epsilon}
$$

and $1+\log \left(N^{2+(1 / n)+\epsilon}\right) \ll N^{\epsilon}$, and this cap will have

$$
|\Delta(r, \mathrm{c})| \gg\left(w_{1}^{2}+\cdots+w_{N}^{2}\right)^{1 / 2} N^{-(1 / 2 n)-2 \epsilon} .
$$

Proof. We note that

$$
E(r, r) \leqq \int_{S}(\sigma \mu(r)+\nu(r, c))^{2} d_{S C}
$$

where $\mu(r)=\mu(C(r, c))$ and $\nu(r, c)=\nu(C(r, c))$. Since $\sigma \mu(r)+\nu(r, c) \ll \sigma$, we have

$$
\begin{aligned}
E(r, r) & \ll \sigma \int_{S}(\sigma \mu(\boldsymbol{r})+\nu(r, \mathbf{c})) d_{S \mathbf{C}} \\
& \ll \sigma^{2} \mu(r) \\
& \ll \sigma^{2} r^{n} .
\end{aligned}
$$

Hence with $\eta=\delta\left(\sigma(2+1 / n+\epsilon) \sigma(1)^{-2-(1 / n)-\epsilon}\right)^{1 / n}$, we obtain

$$
\int_{0}^{\eta} r^{-1} E(r, r) d r \ll \sigma^{2} \eta^{n} \ll \delta^{n} \sigma\left(2+\frac{1}{n}+\epsilon\right) \sigma(1)^{-(1 / n)-\epsilon} .
$$

Therefore if $\delta=\delta(n, \epsilon)>0$ is small, the integral on the left is small in comparison with the integral in (5). Hence for such $\delta$,

$$
\int_{\eta}^{\pi / 2} r^{-1} E(r, r) d r \gg \sigma\left(2+\frac{1}{n}+\epsilon\right) \sigma(1)^{-(1 / n)-\epsilon} .
$$

Now

$$
\int_{0}^{\pi / 2} r^{-1} d r=\log \frac{\pi}{2}-\log \eta \ll 1+\log \frac{\sigma^{2+(1 / n)+\epsilon}}{\sigma\left(2+\frac{1}{n}+\epsilon\right)} .
$$

Thus there is an $r$ with

$$
E(r, r) \gg \sigma\left(2+\frac{1}{n}+\epsilon\right) \sigma^{-(1 / n)-\epsilon}\left(1+\log \left(\frac{\sigma^{2+(1 / n)+\epsilon}}{\sigma\left(2+\frac{1}{n}+\epsilon\right)}\right)\right)^{-1},
$$

and hence a cap $C(r, c)$ with (6). 
2. The method of integral equations. Put

$$
\begin{aligned}
\nu & =0 \quad \text { if } n \text { is even, } \\
& =1 \quad \text { if } n \text { is odd, }
\end{aligned}
$$

and let $\alpha, \beta$ be positive reals with $\alpha<1$ and

$$
\alpha+\beta=1+\nu \text {. }
$$

Put

$$
\begin{aligned}
J= & \int_{0}^{\pi / 2} \int_{r+s \leqq 1}^{\pi / 2} E(r, s) \cos \frac{r-s}{2} \\
& \cdot \cos \frac{r+s}{2}\left(\sin \left|\frac{r-s}{2}\right|\right)^{-\alpha}\left(\sin \frac{r+s}{2}\right)^{-\beta} d r d s .
\end{aligned}
$$

Exactly as in $\$ I V .1$ of [5], one obtains

$$
|J| \ll \int_{0}^{\pi / 2} E(r, r) r^{-1} d r .
$$

Formula (10) in Chapter IV of [5] now becomes

$E(\boldsymbol{r}, s)=\sum_{i=1}^{\infty} \sum_{j=1}^{\infty} w_{i} w_{j}\left(k\left(r, s, w\left(\boldsymbol{p}_{\boldsymbol{i}}, \boldsymbol{p}_{j}\right)\right)-\int_{S} \int_{S} k(\boldsymbol{r}, s, w(\mathbf{x}, \boldsymbol{y})) d_{S} \mathbf{x} d_{S} \boldsymbol{y}\right)$.

Lemma 6 of [5] is valid. The functions $f_{0}(r)$ and $f_{*}(r)$ of $\S I V .4$ of [5] with $\delta=\pi / 2$ satisfy formula (29) in Chapter IV of [5], i.e.

(8) $\int_{0}^{1 / 2} E\left(\frac{\pi}{2} r, \frac{\pi}{2} r\right) f_{0}(r) d r-J=\int_{0}^{1 / 2} E\left(\frac{\pi}{2} r, \frac{\pi}{2} r\right) f_{*}(r) d r$.

Since $f_{0}(r)$ and $f_{*}(r)$ are nonnegative, and since $f_{0}(r) \ll r^{1-p-\alpha}$ and $f_{*}(r) \gg r^{1-n-\alpha}$ as $r \rightarrow 0$, we obtain the following. By (7), the left-hand side of (8) is

$$
\ll \int_{0}^{1 / 2} E\left(\frac{\pi}{2} r, \frac{\pi}{2} r\right) r^{1-\nu-\alpha} d r+|J| \ll \int_{0}^{\pi / 2} E(r, r) r^{-1} d r .
$$

The right-hand side of (8) is

$$
\gg \int_{0}^{1 / 2} r^{1-n-\alpha} E\left(\frac{\pi}{2} r, \frac{\pi}{2} r\right) d r \gg \int_{0}^{\pi / 4}{ }^{1-n-\alpha} E(r, r) d r .
$$

Here $\alpha$ was any fixed number in the interval $0<\alpha<1$. Putting $\epsilon=1-\alpha$ we get 


$$
\int_{0}^{\pi / 2} E(r, r) r^{-1} d r \gg \int_{0}^{\pi / 4} r^{-n+e} E(r, r) d r .
$$

It remains to give a lower bound for the right-hand side of (9). This is where the argument of [5] has to be altered.

\section{Preparations. Write}

$$
k=\{\log \sigma\}
$$

i.e. $k$ is the next larger integer to $\log \sigma$. For any integer $m$, let $P_{m}$ be the subset of $\left\{\boldsymbol{p}_{1}, \boldsymbol{p}_{2}, \cdots\right\}$ consisting of the points $\boldsymbol{p}_{\boldsymbol{i}}$ with

$$
e^{m-1}<w_{i} \leqq e^{m}
$$

and put

$$
\sigma_{m}(\alpha)=\sum_{p_{i} \in P_{m}} w_{i}^{\alpha}
$$

Since $P_{k+1}, P_{k+2}, \cdots$ are empty, we have

$$
\sum_{m=-\infty}^{k} \sigma_{m}(\alpha)=\sigma(\alpha)
$$

Write

$$
A_{m}=e^{m-1}, \quad R_{m}=\frac{1}{2}\left(A_{m} / \sigma\right)^{1 / n}
$$

We have

$$
\left|P_{m}\right|\left(e A_{m}\right)^{\alpha} \geqq \sigma_{m}(\alpha)
$$

where $\left|P_{m}\right|$ denotes the number of elements of $\boldsymbol{P}_{m}$, and observing (12) and putting $\alpha=2+(1 / n)+\epsilon$ we obtain

$$
\sum_{m=-\infty}^{k}\left|P_{m}\right| A_{m}^{2+(1 / n)+\epsilon} \geqq e^{-3} \sigma\left(2+\frac{1}{n}+\epsilon\right) .
$$

The volume $\mu(r)$ of a cap $\mathbf{C}(r, \mathrm{c})$ is of the type

$$
\mu(r)=c_{1} \int_{0}^{r}(\sin \phi)^{n-1} d \phi
$$

and hence one has

$$
c_{2} r^{n-1} \leqq \mu^{\prime}(r) \leqq c_{1} r^{n-1},
$$

where $\mu^{\prime}(r)$ is the derivative of $\mu(r)$. Put

$$
\kappa=\min \left(\frac{1}{3}\left(e^{1 / n}-1\right),\left(6 e c_{1}\right)^{-1}\right) .
$$


Then $R_{m}(1+3 \kappa) \leqq e^{1 / n} R_{m}=R_{m+1}$, and for $m \leqq k$ one has $R_{m}(1+3 \kappa)$ $\leqq R_{k+1} \leqq \frac{1}{2} e^{1 / n}<\pi / 4$. One has

$$
\begin{aligned}
\mu\left(R_{m}(1+3 \kappa)\right)-\mu\left(R_{m}\right) & \leqq 3 R_{m} \kappa c_{1} R_{m}^{n-1}(1+3 \kappa)^{n-1} \\
& <3 e c_{1} \kappa R_{m}^{n}<A_{m} /(2 \sigma)
\end{aligned}
$$

by (13) and (15), and

$$
\mu\left(R_{m}(1+2 \kappa)\right)-\mu\left(R_{m}(1+\kappa)\right) \geqq R_{m} \kappa c_{2} R_{m}^{n-1}=c_{3} R_{m}^{n} .
$$

4. Caps with given center. Let a point $\mathrm{c}$ on $S$ be fixed, and let $m \leqq k$ be fixed. Assume that precisely

$$
h=h_{m}(\mathrm{c})>0
$$

of the points of $P_{m}$ lie in $\mathbf{C}\left(R_{m}(1+2 \kappa), \mathrm{c}\right)$ but not in $\mathbf{C}\left(R_{m}(1+\kappa), \mathrm{c}\right)$, i.e. in the complement of $\mathbf{C}\left(R_{m}(1+\kappa), \mathrm{c}\right)$ in $\mathbf{C}\left(R_{m}(1+2 \kappa), \mathrm{c}\right)$. Then for any $r$ in the interval $R_{m} \leqq r<R_{m}(1+\kappa)$, one has $\nu\left(r+2 R_{m} \kappa, \mathrm{c}\right)$ $-\nu(r, \mathrm{c}) \geqq h A_{m}$. Hence since $R_{m}(1+3 \kappa) \leqq R_{m+1}$, we have

$$
\begin{aligned}
& \int_{R_{m}}^{R_{m}+1} r^{-n+n \epsilon}(\sigma \mu(r)-\nu(r, \mathrm{c}))^{2} d r \\
& \gg \int_{R_{m}}^{R_{m}(1+\kappa)} r^{-n+n \epsilon}\left((\sigma \mu(r)-\nu(r, \mathrm{c}))^{2}+\left(\sigma \mu\left(r+2 R_{m} \kappa\right)-\nu\left(r+2 R_{m} \kappa, \mathrm{c}\right)\right)^{2}\right) d r \\
& \gg \int_{R_{m}}^{R_{m}(1+\kappa)} r^{-n+n \epsilon}\left(\nu\left(r+2 R_{m} \kappa, \mathrm{c}\right)-\nu(r, \mathrm{c})-\sigma\left(\mu\left(r+2 R_{m} \kappa\right)-\mu(r)\right)\right)^{2} d r \\
& \gg \int_{R_{m}}^{R_{m}(1+\kappa)} r^{-n+n \epsilon}\left(h A_{m}-\frac{1}{2} A_{m}\right)^{2} d r
\end{aligned}
$$

by (16). We therefore get

$$
\begin{aligned}
& \gg h^{2} A_{m}^{2} \int_{R_{m}}^{R_{m}(1+\kappa)} r^{-n+n \epsilon} d r \\
& \gg h A_{m}^{2} R_{m}^{1-n+n \epsilon} .
\end{aligned}
$$

5. End of the proof. We have $h=h_{m}(\mathrm{c})$, and

$$
\int_{S} h_{m}(\mathrm{c}) d \mathrm{c}=\left|P_{m}\right|\left(\mu\left(R_{m}(1+2 \kappa)\right)-\mu\left(R_{m}(1+\kappa)\right)\right) \gg\left|P_{m}\right| R_{m}^{n}
$$

by (17). It follows that 


$$
\begin{aligned}
\int_{R_{m}}^{R_{m}+1} r^{-n+1+\epsilon} E(r, r) d r & \geqq \int_{R_{m}}^{R_{m+1}} r^{-n+1+n \epsilon} E(r, r) d r \\
& \gg\left|P_{m}\right| R_{m}^{n} A_{m}^{2} R_{m}^{1-n+n \epsilon} \gg\left|P_{m}\right| A_{m}^{2+(1 / n)+\epsilon} \sigma^{-(1 / n)-\epsilon} .
\end{aligned}
$$

Therefore

$$
\begin{aligned}
& \int_{0}^{\pi / 4} r^{-n+1+\epsilon} E(r, r) d r \gg\left(\sum_{m=-\infty}^{k}\left|P_{m}\right| A_{m}^{2+(1 / n)+\epsilon}\right) \sigma^{-(1 / n)-\epsilon} \\
& \gg \sigma\left(2+\frac{1}{n}+\epsilon\right) \sigma^{-(1 / n)-\epsilon}
\end{aligned}
$$

by (10). In view of (9), the theorem follows.

\section{REFERENCES}

1. G. H. Hardy, J. E. Littlewood and G. P6lya, Inequalities, Cambridge Univ. Press, New York, 1934. MR 13, 727.

2. K. F. Roth, On irregularities of distribution, Mathematika 1 (1954), 73-79. MR 16, 575.

3. W. M. Schmidt, Irregularities of distribution. II, Trans. Amer. Math. Soc. 136 (1969), 347-360. MR 38 \#3237.

4. - Irregularities of distribution. III, Pacific J. Math. 29 (1969), 225-234.

5. —- Irregularities of distribution. IV, Invent. Math. 7 (1969), 55-82.

University of Colorado, Boulder, Colorado 80302 